\title{
Kurze Mitteilung über zwei für die Deutsche Bucht neue Polychätenarten der Gattungen Capitomastus und Goniada
}

\author{
Von Erich Ziegelmeier \\ Biologische Anstalt Helgoland, List auf Sylt \\ Forschungsinstitut der Bundesanstalt für Fischerei \\ (Mit 1 Abbildung und 2 Tabellen)
}

Bei den seit dem Herbst 1949 wieder aufgenommenen quantitativen Bodengreiferuntersuchungen in der Deutschen Bucht, über deren Ergebnisse in Kürze ausführlich berichtet wird, konnten zwei für dieses Gebiet bisher noch nicht nachgewiesene Polychätenarten festgestellt werden:

1. Capitomastus minimus (Langerhans). Von diesem Capitelliden wurde ein Exemplar in einer 1/10-qm-Bodengreiferprobe bei Station Hever III (Position: $54^{0} 25,4^{\prime} \mathrm{N}-8^{0} 4,5^{\prime} \mathrm{O}, 18 \mathrm{~m}$ Tiefe, reiner, feiner Sand) am 7.9. 1951 gefunden. Die Länge des Wurmes beträgt $11 \mathrm{~mm}$, die durchschnittliche Dicke $\left.0,75 \mathrm{~mm}^{1}\right)$. Gut erkenntlich ist die Art an den drei nur Haarborsten tragenden vordersten Segmenten, wodurch sich Capitomastus deutlich von den Arten der nahe verwandten Gattungen Capitella und Capitellides unterscheidet, bei denen die sechs ersten Segmente Haarborsten besitzen. Fauver gibt als Fundorte Atlantik (Madeira) und Mittelmeer (Neapel) an.

Wahrscheinlich ist die Art in unserem Gebiet bisher übersehen worden. Es läßt sich also nicht feststellen, ob Capitomastus auch zu den Arten gehört, die infolge der Erwärmung der nordatlantischen Gewässer in die Nordsèe eingewandert sind.

2. Im Frühjahr und Herbst 1952 trat in den Bodengreiferproben von zwei Stationen westlich Sylt (Tab. 1), besonders häufig im groben Sand, eine Goniada auf, die sich von den in unseren Meeresteilen bisher nachgewiesenen Spezies wesentlich unterscheidet, aber mit der von Rioja (1923) neu aufgestellten Goniada galaica (Fundort: An der galicischen Atlantikküste, Spanien) in den für die Artdiagnose wichtigen Merkmalen ziemlich übereinstimmt mit Ausnahme der Parapodien und der Rüsselbewaffnung (Tab. 2).

Insgesamt konnten 101 Exemplare aus den Fängen herausgesucht werden, in denen neben Goniada oft zahlreich Praegeria remota (Southern), Staurocephalus kefersteini (McINT.), Aonides paucibranchiata (Southern),

1) Herrn Prof. H. Friedkich-Bremerhaven möchte ich an dieser Stelle tür die Nạchbestimmung bestens danken. 
Spio filicornis (O. F. MülLer) und bei Westerland I im Herbst 1952 Polygordius appendiculatus (FraIPONT) gefunden wurden.

Tabelle 1. Vorkommen von Goniada spec.

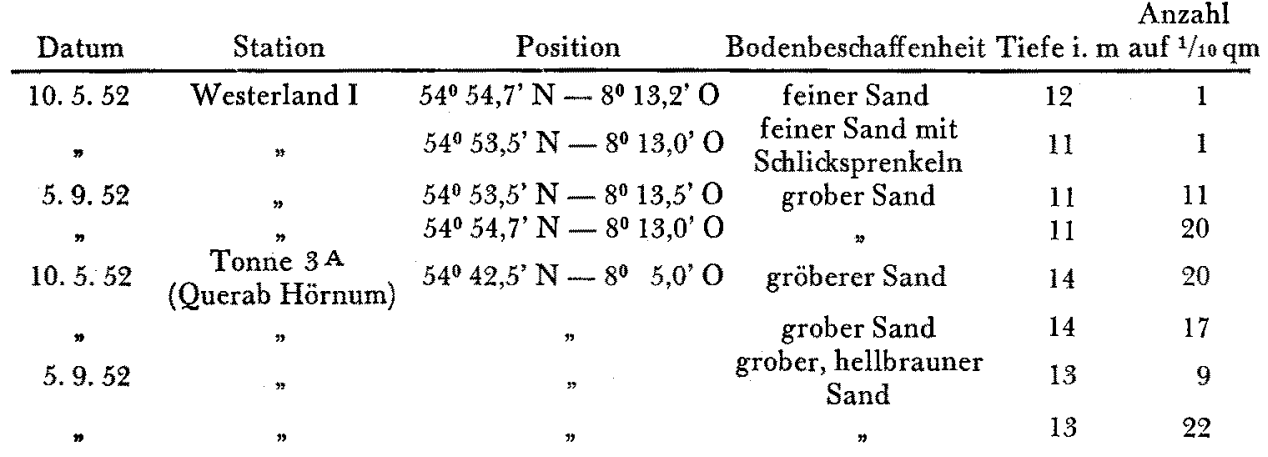

Tabelle 2

Zusammenstellung der wichtigsten Merkmale für die in den europäischen Meeresteilen gefundenen Arten der Gattung Goniada außer G. pallida - von ARwidsson 1899 nach einem unvollständigen Exemplar (Fundort: Bohuslän) beschrieben, nach FAUvet und StøpBowirz aber mit $G$, norvegica identisch - G. felicissima KingBerg (Fundort: bei St. Helena) und G. bobrezkii Annenkova (Fundort: Schwarzes Meer).

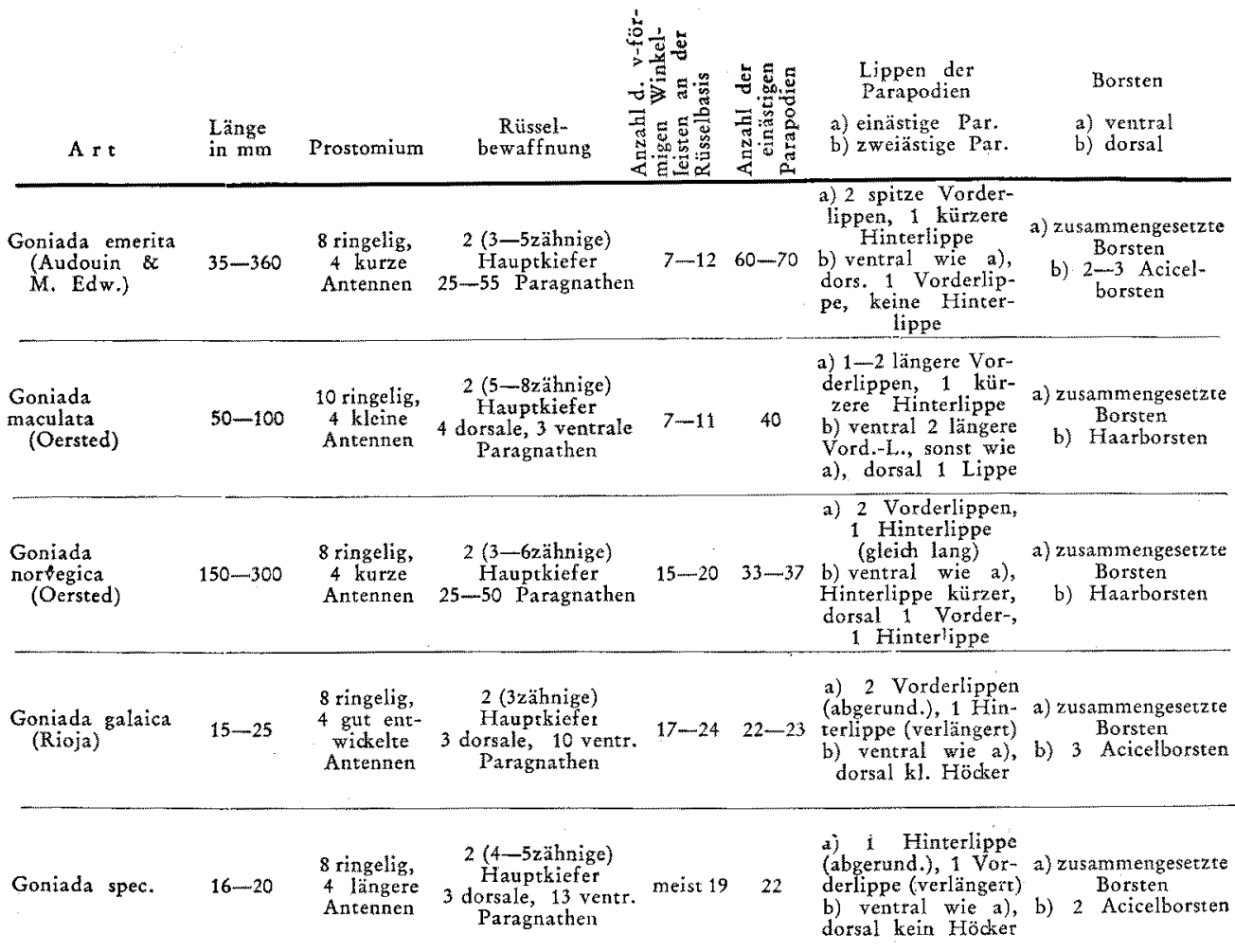

Zur Vervollständigung der in Tab. 2 angegebenen Merkmale sollen die hier festgestellten Tiere im folgenden kurz beschrieben werden:

Die Länge beträgt $16-20 \mathrm{~mm}$, die Breite (ohne Parapodien) im thorakalen Abschnitt 0,30-0,35 $\mathrm{mm}$ und in der Körpermitte 0,4 mm. 80-100 
Segmente, Körper hinter der rundlichen vorderen Region nur schwach dorsoventral abgeplattet. Die mit Formalin fixierten Tiere sind blaßgelb, der mit einästigen Parapodien versehene Vorderabschnitt heller und durchscheinender. Vom 23. Segment an sind ventral einzelne sehr kleine, rötlich-braune Punkte zu erkennen, die in der Mitte auf der Grenzlinie zwischen zwei Segmenten liegen.

Am vorderen Ende des schlanken 8ringeligen Prostomiums, das eine durchschnittliche Länge von $0,6 \mathrm{~mm}$ hat, stehen vier längere Tentakel, die, unregelmäßig knotenartig verdickt, zu dünnen, parallelwandigen Spitzen auslaufen, deren Längen etwa $1 / 4$ der Gesamtlänge eines Tentakels betragen (Abb. G). Am Grunde des breiteren Basalteils des Prostomiums, der zwei Augen besitzt, lassen sich deutlich zu beiden Seiten zwei kurze cirrusartige Anhänge erkennen, die wohl den von Moore $(1903,1905)$ bei Goniada annulata und G. foliacea, von Vort (1911) bei Goniada maculata und von StøpBowitz (1941) sowie Keferstein (1863) bei Glycera capitata erwähnten Palpen gleichzusetzen sind.

Der mit sehr kleinen Papillen besetzte Rüssel trägt an der Basis lateral je meist $19 \mathrm{v}$-förmige Winkelleisten (Abb. F). Der vordere Rand des Rüssels wird aus einem Kranz von 18 halbkugeligen großen Papillen gebildet. Zwischen den vier- (zuweilen fünf-)zähnigen Hauptkiefern (Abb. D) stehen dorsal drei Paragnathen, und ventral schließen sich 13 Nebenkiefer an, die mit den dorsalen einen geschlossenen Kreis bilden.

Sämtliche Paragnathen bestehen aus zwei Stücken, dem Hauptteil und dem Kieferstückchen. Der $\mathrm{x}$-förmige Hauptteil ist mit seinen zwei oberen Schenkeln in der Wand des Rüssels eingewachsen (Abb. E), und seine beiden unteren Schenkel bilden zwei spitze, freistehende Zähnchen. Das drei- oder zweizähnige kleine Kieferstück ist scharnierartig so eingehängt, daß die beiden Teile gegeneinander beweglich sind. Im ganzen ragen also fünf bzw. vier Zähnchen aus der Rüsselwandung heraus²).

Die drei dorsalen Paragnathen der hier gefundenen Goniada haben dreizähnige, die 13 ventralen zweizähnige Kieferstücke. Bei den 13 ventralen können deutlich neun, etwa in der Größe der dorsalen, von vier kleinen Nebenkiefern unterschieden werden. Zwei kleine liegen neben den Hauptkiefern, es folgen ventral zu beiden Seiten drei große, dann wieder zwei kleine, zwischen denen wiederum drei große Paragnathen stehen. Dadurch ergibt sich der bereits erwähnte geschlossene Kreis, bei dem die vier kleinen so verteilt sind, daß zwischen ihnen stets drei große Nebenkiefer liegen. Die Haupt- und Nebenkiefer sind dunkelbraun bis schwarz gefärbt, sie lassen sich auch bei nicht ausgestülptem Rüssel gut erkennen.

Alle Parapodien besitzen im Gegensatz zu den Rudern von Goniada galaica nur eine abgerundete, Hinterlippe (wie Goniadella gracilis Verrill, vgl. O. Hartmann 1950, S. 42) und eine sie weit überragende, spitzauslaufende Vorderlippe (Abb. A bis C). Ventral- und Dorsalcirrus sind gut entwickelt. In den beiden über und unter der Stützborste (Acicula) stehenden

2) Aus der Zeichnung von Rroja (1923, S. 339, Fig. 2 C) eines dorsalen Paragnathen von Goniada galaica, bei dem das dreizähnige kleine Kieferstück mit seinem mittelsten Zähnchen hinter den $x$-förmigen Hauptteil greift, geht der Bau der zusammengesetzten Nebenkiefer nicht deutlich hervor. Wahrscheinlich ist bei der Präparation, vielleicht auch eine durch Alkoholfixierung verursachte Verlagerung eingetreten. Die von mir beobachtete Lage der Teilstücke wurde bei Formalin-fixierten Tieren festgestellt. 


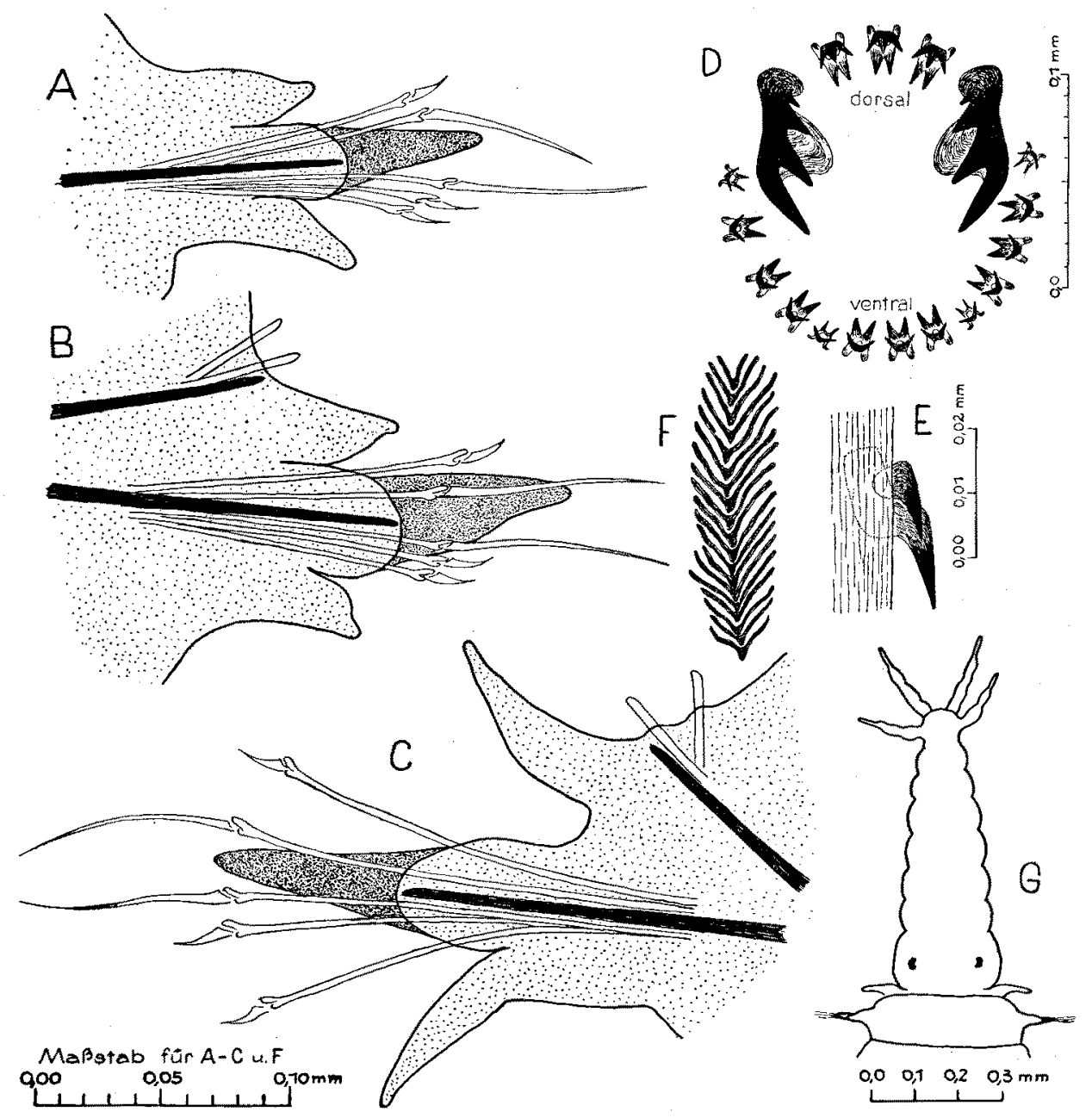

Abb. 1. $A=10$. Parapodium $-B=24$. Parapodium $-\mathrm{C}=$ Parapodium aus der hinteren Körperregion (bei $\mathrm{A}$ bis $\mathrm{C}$ die Vorderlippe dunkel punktiert) $-\mathrm{D}=$ Rüsselbewaffnung (Hauptund Nebenkiefer) $-E=$ zusammengesetzter Paragnath von der Seite, in der Rüsselwandung sitzend $-\mathrm{F}=\mathrm{v}$-förmige Winkelleisten an der Rüsselbasis $-\mathrm{G}=$ Prostomium. (gezeichnet nach Formalin-fixierten Tieren)

Bündeln mit je 2-4 heterogomphen Borsten tragen die der Stützborste am nächsten liegenden längere Endglieder, die bisweilen in der Körpermitte sehr lang sein können (Epitokie?). Die äußeren Borsten haben stets kurze Endglieder. Die vom 23. Segment ab beginnenden zweiästigen Parapodien unterscheiden sich von den einästigen dadurch, daß über dem Dorsalcirrus eine Stützborste und zwei kurze, dicke, immer über der Acicula liegende einfacke Borsten den dorsalen Ast bilden. Lippen und Cirren konnten in dem reduzierten Dorsalast nicht beobachtet werden, wenn nicht der Dorsalcirrus der einästigen Parapodien, der sich nach dem Körperende zu immer weiter von den Lippen des ventralen Astes absetzt, als Ventralcirrus des Notopodiums gedeutet wird.

Zwei Analcirren in der Länge von etwa zwei Segmenten aus der Körpermitte. 
Vermutlich ist auch Goniada spec. bisher übersehen worden. Die dünnen, fädigen Würmer sind beim Aussuchen der Fänge an Bord in den Sieben meist schwer zu erkennen, da sie an leeren Muschelschalen, Schneckengehäusen und anderen Bestandteilen des Siebrestes, schließlich am Maschendraht der Siebe festkleben. Eine bei den Bodengreiferarbeiten jetzt angewandte Methode, bei der der gesamte Siebinhalt nach dem Durchspülen konserviert wird, ermöglicht, daß die im Siebsatz zurückgebliebenen Tiere nach einer Untersuchungsfahrt im Laboratorium restlos herausgesucht und dann bestimmt werden können.

\section{Literatur}

F a uve 1, P., Polychètes errantes. Faune de France 5, Paris, 1923.

- Polychètes sedentaires. Faune de France 16, Paris, 1927.

Friedrich, H., Polychaeta. Tierw. N. u. Ostsee. Teil VI b, 1938.

Hartmann, Olga, Goniadidae, Glyceridae and Nephthyidae, Allan Hancock Pacific Exped. 15, Nr. 1, 1950.

Keferstein, W., Untersuchungen über Niedere Seethiere. Zeitschr. f. wiss. Zool. 12, 1863.

Moore, J. P., Polychaeta from the Coastal Slope of Japan etc. Proc. Acad. Nat. Sci. Philadelphia, 1903.

- New Species of Polychaeta from the North Pacific. Ebenda, 1905.

Rioja, E., Algunas especies de Anelidos Poliquetos de las costas de Galicia. Bol. R. Soc. esp. Hist. Nat., XXIII, Madrid, 1923.

Støp-B owitz, C., Les Glycériens de Norvège. Medd. zool. Mus. Osio, No. 51, 1941.

Voit, M., Dic Glyceriden der Nordsee. Wiss. Meeresunt., Abt. Kiel, NF. 13, 1911.

\section{Nachtrag bei der Korrektur}

In der Zusammenstellung (Tabelle 2) auf Seite 256 der vorliegenden kurzen Mitteilung sind Goniada felicissima Kingrerg und Goniada bobrezkii Annenkova nicht berücksichtigt worden, da nach den Angaben von O. Hartmann (1950 - S. 14) bei der Einteilung der Goniadidae nach der notopodialen Borstentracht die beiden Spezies zu der Gruppe gerechnet wurden, bei der das Aussehen der Borsten des dorsalen Astes nicht bekannt ist. Es wurde angenommen, daßs der Verfasserin die Originalliteratur vorgelegen hat. Während der Drucklegung der vorangegangenen Darstellung konnte der zuerst nicht zugängliche Beitrag: ANNENkova, N. Nachtrag zur Polychaetenfauna des Schwarzen Meeres. I. Goniada bobrezkii sp. n. in Comptes Rendus de l'Académie des Sciences de 1'URSS, Leningrad 1929, S. 495-497, erst jetzt eingesehen werden. Dabei stellte sich heraus, daß die aus der Deutschen Bucht beschriebene Goniada in den wichtigsten Merkmalen mit Goniada bobrezkii (AnNEnKova) bis auf die folgenden geringfügigen Abweichungen übereinstimmt:

\section{Goniada spec.}

16 Paragnathen

22 einästige Parapodien

1 Paar Augen an der Basis des

Prostomiums

Meist $19 \mathrm{v}$-förm. Winkelleisten

Ab 23. Segment ventral kleine rötlichbraune Punkte zwischen den Segmenten

\section{Goniada bobrezkii}

\section{Paragnathen}

24 einästige Parapodien

2 Paar Augen (1 Paar stets an der Basis des Prostomiums, je 1 Auge auf dem 2. und 3. bzw. 3. und 4. Ring des Prostomiums)

17-18 v-förm. Winkelleisten

Segmente des hinteren Körperabschnittes mit braunen Pigment-Querstreifen

Als Fundorte der beiden, der Artbeschreibung zugrundeliegenden Exemplare von Goniada bobrezkii gibt Annenrova an: Schwarzes Meer, Südküste der Krim beim Kloster Georgievskij, Grund: Sand mit Kieselsteinen, Tiefe $=12,5 \mathrm{~m}$ und Bucht am Kap Ilja, Grund: Sand aus Muschelfragmenten, Tiefe $=17 \mathrm{~m}$.

Nach der Abbildung des Parapodiums und der Artdiagnose von Annenkova ist sehr wahrscheinlich, dał die hier gefundene Goniada mit der von Annenrova ,als neue und einstweilen endemische Art des Schwarzen Meeres" angesehenen Goniada bobrezkii identisch ist. 